\title{
Ortholord Tablets: Nutritional Support for Rheumatoid Arthritis
}

\section{Dr. Sampoorna, Madugula Mahender, S. Vijaya Bhavani \& Bhavya Krishna Ponnakanti}

Besiara Pharmaceuticals Makers of ORTHOLORD Tablets 4-77/2, Badangpet, Balapur (M), Hyderabad, India.

DOI: $10.46382 / M J B A S .2020 .4204$

Rheumatoid Arthritis (RA) is a chronic systemic autoimmune disease that primarily affects the lining of the synovial joints and is associated with progressive disability, premature death, and socioeconomic burdens. The aim of the study was to evaluate the clinical efficacy and safety of glucosamine phosphate, methylsulfonylmethane (MSM), Vitamin C, Curcuma, in the management of Rheumatoid Arthritis. All those patients fulfilling the inclusion criteria presenting with Rheumatoid Arthritis to emergency department or to the out-patient department of general medicine were enrolled in the study. It included 180 adult patients with Rheumatoid Arthritis of both the sexes. All the participants were evaluated by detailed history, clinical examination and severity assessment. Cases were excluded if there was insufficient clinical information to permit allocation of a priority level at diagnosis. Patients aged 25 years or above referred or diagnosed with Rheumatoid Arthritis at our outpatient clinic were included in the study. All together 180 patients were participated in this study, and the response was $100 \%$. All families were approached.

Glucosamine sulfate might provide some pain relief for people with Rheumatoid Arthritis. Glucosamine has been found to have positive effects on pain treatment \& Relieving pain and improving physical function. MSM may help to relieve joint soreness, stiffness and swelling. MSM is to decrease joint or muscle pain. Collagen peptide significantly reducing pain and joint inflammation.

Keywords: Rheumatoid Arthritis, Early Treatment, Inflammatory Disorder, Joints.

\section{INTRODUCTION}

\section{Rheumatoid Arthritis (RA)}

Rheumatoid Arthritis (RA) is an autoimmune disease that can cause joint pain and damage throughout your body. The joint damage that RA causes usually happens on both sides of the body. So, if a joint is affected in one of your arms or legs, the same joint in the other arm or leg will probably be affected, too. This is one way that doctors distinguish RA from other forms of arthritis, such as osteoarthritis (OA).
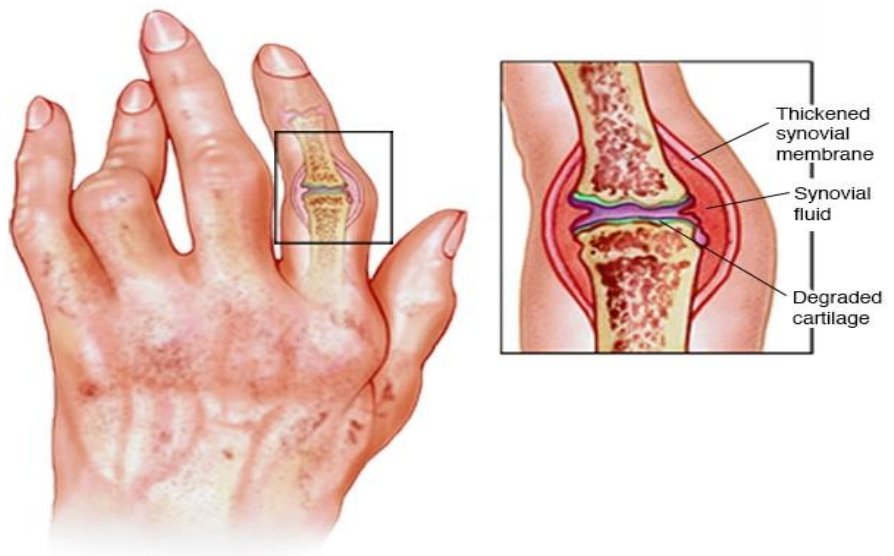

In some people, the condition can damage a wide variety of body systems, including the skin, eyes, lungs, heart and blood vessels. An autoimmune disorder, Rheumatoid Arthritis 
occurs when your immune system mistakenly attacks your own body's tissues. Rheumatoid Arthritis can cause pain, swelling and deformity. As the tissue that lines your joints (synovial membrane) becomes inflamed and thickened, fluid builds up and joints erode and degrade.

\section{Symptoms}

Signs and symptoms of Rheumatoid Arthritis may include:

$\checkmark$ Tender, warm, swollen joints

$\checkmark$ Joint stiffness that is usually worse in the mornings and after inactivity

$\checkmark$ Fatigue, fever and loss of appetite

$\checkmark$ Joint pain

$\checkmark$ Joint redness

$\checkmark$ Weight loss

$\checkmark$ Unexplained weight loss, which is possibly an indirect effect of inflammation

$\checkmark$ Joint warmth: Joint warmth is caused by inflammation and may be present before redness or swelling occurs.

$\checkmark$ Numbness and tingling

Early Rheumatoid Arthritis tends to affect your smaller joints first particularly the joints that attach your fingers to your hands and your toes to your feet. As the disease progresses, symptoms often spread to the wrists, knees, ankles, elbows, hips and shoulders. In most cases, symptoms occur in the same joints on both sides of your body. About 40 percent of the people who have Rheumatoid Arthritis also experience signs and symptoms that don't involve the joints. Rheumatoid Arthritis can affect many non-joint structures, including Skin, Lungs, Nerve tissue, Bone marrow, Blood vessels.

\section{Causes}

Rheumatoid Arthritis is an autoimmune condition, which means it's caused by the immune system attacking healthy body tissue. However, it's not yet known what triggers this. Your immune system normally makes antibodies that attack bacteria and viruses, helping to fight infection. Rheumatoid Arthritis occurs when your immune system attacks the synovium- the lining of the membranes that surround your joints. The resulting inflammation thickens the synovium, which can eventually destroy the cartilage and bone within the joint. The tendons and ligaments that hold the joint together weaken and stretch. Gradually, the joint loses its shape and alignment. While your genes don't actually cause Rheumatoid Arthritis, they can 
make you more susceptible to environmental factors such as infection with certain viruses and bacteria that may trigger the disease.
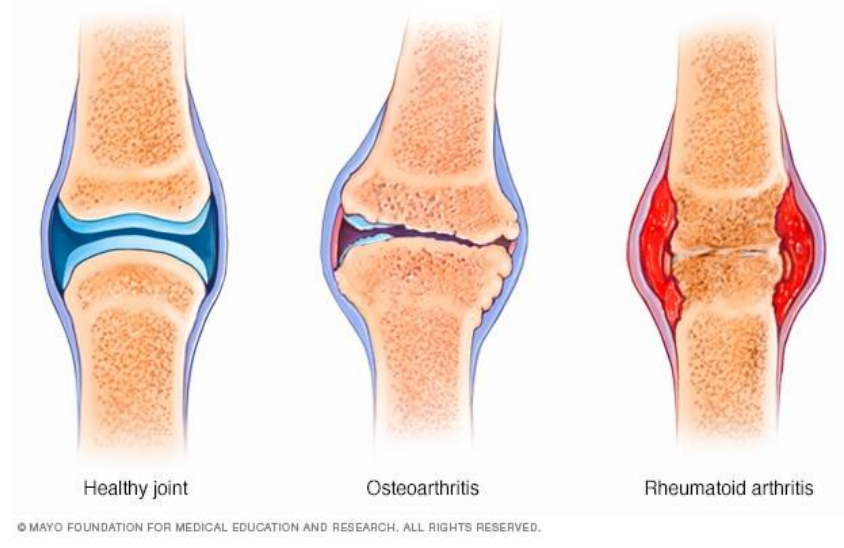

\section{Rheumatoid Arthritis vs. osteoarthritis}

Osteoarthritis, the most common form of arthritis, involves the wearing away of the cartilage that caps the bones in your joints. With Rheumatoid Arthritis, the synovial membrane that protects and lubricates joints becomes inflamed, causing pain and swelling.

\section{Pathophysiology}

Rheumatoid Arthritis (RA) is a chronic autoimmune disease. When the immune system is functioning normally, it recognizes things like harmful bacteria and viruses, and responds by creating an "army" of antibodies that seek out and fight them off. In an autoimmune disease, the body mistakenly thinks that normal tissues or organs of the body are harmful, leading to inflammation and damage. In RA, this effectively results in the body's immune system attacking the tissues of the joints, causing pain and inflammation. RA can cause permanent damage to joints. Whilst the cause of RA is not fully understood, a number of risk factors such as smoking, obesity, and family history have been implicated in the development of RA. The combination of these factors can send a trigger to the body to create antibodies known as autoantibodies that seek out joint linings.

These autoantibodies include rheumatoid factor (RF) and anti-cyclic citrullinated peptide antibody (anti-CCP). There is an increased number of both type synoviocytes and is infiltrated with immune and inflammatory cells: particularly macrophages, B- and T-lymphocytes, plasma cells and dendritic cells. Synovial cell hyperplasia and endothelial cell activation are early events in the pathologic process that progresses to uncontrolled inflammation and consequent cartilage and bone destruction. Genetic factors and immune system abnormalities contribute to disease propagation 


\section{Inflammation}

This results in the production of chemicals being released including tumor necrosis factor alpha (TNF-a), Interleukin (IL)-1, IL-6, IL-8, transforming growth factor beta (TGF- $\beta$ ), fibroblast growth factor (FGF) and platelet-derived growth factor (PDGF).

\section{Joint and tissue destruction}

These chemicals inflame and damage the body's cartilage, bone, tendons, and ligaments, resulting in the symptoms seen in Rheumatoid Arthritis.

\section{Risk Factors}

Age: Rheumatoid Arthritis can occur at any age, but it most commonly begins in middle age. Your sex: Women are more likely than men to develop Rheumatoid Arthritis.

Family history: If a member of your family has Rheumatoid Arthritis, you may have an increased risk of the disease.

Smoking Cigarette: smoking increases your risk of developing Rheumatoid Arthritis

Environmental exposures: Exposures such as asbestos or silica may increase the risk of developing Rheumatoid Arthritis.

Obesity: People especially women age 55 and younger who are overweight or obese appear to be at a somewhat higher risk of developing Rheumatoid Arthritis.

\section{Complications}

Rheumatoid Arthritis increases your risk of developing:

$>$ Osteoporosis

$>$ Rheumatoid nodules

$>$ Dry eyes and mouth

$>$ Infections

> Abnormal body composition

$>$ Heart problems

$>$ Lung disease

\section{Diagnosis}

Rheumatoid Arthritis can be difficult to diagnose in its early stages because the early signs and symptoms mimic those of many other diseases. There is no one blood test or physical finding to confirm the diagnosis. During the physical exam, your doctor will check your joints 
for swelling, redness and warmth. He or she may also check your reflexes and muscle strength.

Blood tests: People with Rheumatoid Arthritis often have an elevated erythrocyte sedimentation rate (ESR, or sed rate) or C-reactive protein (CRP), which may indicate the presence of an inflammatory process in the body. Other common blood tests look for rheumatoid factor and anti-cyclic citrullinated peptide (anti-CCP) antibodies.

Imaging tests: Your doctor may recommend X-rays to help track the progression of Rheumatoid Arthritis in your joints over time. MRI and ultrasound tests can help your doctor judge the severity of the disease in your body.

\section{Lifestyle Modifications}

$\checkmark$ Exercise regularly

$\checkmark$ Apply heat or cold

$\checkmark$ Rest: If you're experiencing a flare-up in symptoms, it's important to rest and give your joints a chance to recover

$\checkmark \quad$ Limit Alcohol Intake

$\checkmark$ Quit Smoking

\section{AIM AND OBJECTIVES}

Aim: The aim of the study was to evaluate the clinical efficacy and safety of glucosamine phosphate, methylsulfonylmethane (MSM), Vitamin C, Curcuma, in the management of of Rheumatoid Arthritis.

Objectives: To determine the Clinical Efficacy and Safety of Glucosamine, Hyaluronic acid, Collagen Peptides, boswellia serata in the management Osteoarthritis.

\section{LITERATURE REVIEW}

James T Birch Jr, et al, (2010): Rheumatoid Arthritis is an inflammatory disease of the joints causing pain and stiffness, pathologically characterized by chronic synovitis. Without proper treatment, it progresses to cause joint deformity that result in significant loss of function. Extra-articular disease can also occur, which exacerbates morbidity and mortality associated with the disease. Patients from all age groups can acquire the disease, hence the additional categories of juvenile onset and elderly onset Rheumatoid Arthritis. Disease-modifying anti rheumatic drugs are the mainstay of therapy, and should be initiated as early as possible in the course of the disease in consultation with a rheumatologist. 
William G Dixon, et al, (2005): The 'life cycle' of established Rheumatoid Arthritis can be divided into four phases. The first is the period leading up to the onset of arthritis. The second is the period during which persistence or remission is determined.

The third is the evolution into a specific form of inflammatory arthritis, and the fourth is the outcome/severity of that arthritis. In some patients, these four phases follow in rapid succession; however, in other patients, the time course is prolonged over several months or years. This chapter explores the hypothesis that these four phases are distinct in the majority of patients, and that different genetic and environmental factors influence the various phases.

It investigates the suggestion that a defect in the hypothalamic-pituitary-adrenal axis may underlie the persistence of inflammatory arthritis.

\section{DRUG PROFILE}

Glucosamine Sulfate \& Chondroitin Sulfate Sodium: Glucosamine and Chondroitin Sulfate are compounds that work together to treat inflammation due to arthritis as found in RA and osteoarthritis. Glucosamine is key to cartilage formation and maintenance and is manufactured from shellfish. Chondroitin is also a key component to cartilage maintenance as its main function is to attract water to the cartilage to provide better shock absorption during physical activity. For RA sufferers, Glucosamine has been found to have positive effects on pain treatment. Chondroitin Sulfate inhibits inflammatory enzymes from entering the synovial fluid, which is key to minimizing the destruction of cartilage in autoimmune disorders, such as Rheumatoid Arthritis. Glucosamine and Chondroitin Sulfate are used to build up cartilage between the joints.

\section{Methylsulfonylmethane (MSM):}

MOA: MSM may help to relieve the pain and inflammation of Rheumatoid Arthritis: MSM has natural anti-inflammatory properties that may help to relieve joint soreness, stiffness and swelling. MSM is to decrease joint or muscle pain. Studies have shown that MSM significantly reduces inflammation in your body. It also inhibits the breakdown of cartilage, a flexible tissue that protects the ends of your bones in joints.

Collagen Peptide: It's rich in amino acids that play an important role in the building of joint cartilage and it may have anti-inflammatory effects. Studies into the role of collagen in treating Rheumatoid Arthritis suggest that it doesn't have a significant effect in reducing pain and joint inflammation. 
MOA: The peptide also increased anti-inflammatory cytokines as well as an inhibiting proinflammatory cytokines. In fact, arthritis induced by collagen is an example of an autoimmune disease mediated by proinflammatory cytokines.

\section{Hyaluronic Acid}

MOA: HA can regulate the growth and function of chondrocytes by binding to CD44 receptors on the chondrocytes. Synovitis is often found in patients with Rheumatoid Arthritis (RA) and is supposed to result from CD44 activity. HA control inflammation and synovitis in the foot and ankle joints caused by Rheumatoid Arthritis.

Vitamin $C$ The effect of intravenous vitamin C (IVC) treatment on subjects with RA demonstrated that IVC therapy with dosages of $7.5 \mathrm{~g}-50 \mathrm{~g}$ can reduce inflammation and the pain levels," the researchers wrote. "The inflammation as measured by C-reactive protein levels was decreased on average by $44 \%$. Vitamin C might protect against the development of osteoarthritis, Rheumatoid Arthritis, and inflammatory polyarthritis, according to some studies.

Boswellia: It is effective in reducing inflammation, so it may also lessen the symptoms of RA. The Arthritis Foundation recommends boswellic acid capsules as a potential therapy for RA, as well as for osteoarthritis.

Curcumin: Some research suggests that the spice turmeric may help relieve the inflammatory symptoms of this condition. ... In particular, turmeric contains a compound called curcumin, which has anti-inflammatory properties that may benefit people with rheumatoid arthritis (RA)

\section{MATERIALS AND METHODS}

The associations between older age, obesity and increased rates of knee RA are well understood. Participants were aged 25 to 75 years at baseline of Rheumatoid Arthritis. Subjects were identified by random-digit dialing, and interviews were conducted by trained bilingual research assistants. The telephone response rate was $91 \%$. Subjects were recruited from the telephone sample to have an in-person baseline interview and assessment. On enrollment, all patients underwent complete physical examination. Informed written consent was signed from all participants. Data were collected through interviews, Physical examinations were conducted. All those patients fulfilling the inclusion criteria presenting with Rheumatoid Arthritis to emergency department or to the out-patient department of general medicine were enrolled in the study. It included 180 adult patients with Rheumatoid Arthritis of both the sexes. All the participants were evaluated by detailed 
history, clinical examination and severity assessment. Cases were excluded if there was insufficient clinical information to permit allocation of a priority level at diagnosis. We excluded patients aged 80 years or above, with a history of major illness like liver, renal or lung diseases, those taking vitamin supplements or calcium or on anticonvulsants, diabetes mellitus, alcoholism, history of pulmonary surgery or tuberculosis, or a psychiatric history and pregnancies.

Patients aged 25 years or above referred or diagnosed with Rheumatoid Arthritis at our outpatient clinic were included in the study.

\section{Statistical Analysis:}

Paired t-test was used to assess statistical significance. A level of $p<0.005$ was accepted as statistically significant. Chi square test for categorical variables.

\section{RESULTS AND DISCUSSION}

A total of 200 patients were enrolled as per inclusion and exclusion criteria, All together 180 patients were participated in this study, and the response was $100 \%$. All families were approached. Only one family of four adults refused to participate in the study.

They were not included in the study. Table 1 shows the participants were divided into 4 groups by age: 20-30years ( $n=52,28.8 \%), 31-40$ years $(n=48,26.6 \%), .41-50$ years $(n=11,41.22 .7 \%), 51-60$ years $(n=39,21.6 \%)$.

The majority of patients in the age group between 20 -30years $(n=52,28.8 \%)$.

Table 1

Age wise distribution of patients

\begin{tabular}{|c|c|c|}
\hline $\mathrm{N}=180$ & $\begin{array}{c}\text { No. of } \\
\text { Patients }\end{array}$ & Percentage \\
\hline $20-30 \mathrm{yrs}$ & 52 & $28.8 \%$ \\
\hline $31-40$ & 48 & $26.6 \%$ \\
\hline $41-50$ & 41 & $22.7 \%$ \\
\hline $51-60$ & 39 & $21.6 \%$ \\
\hline
\end{tabular}




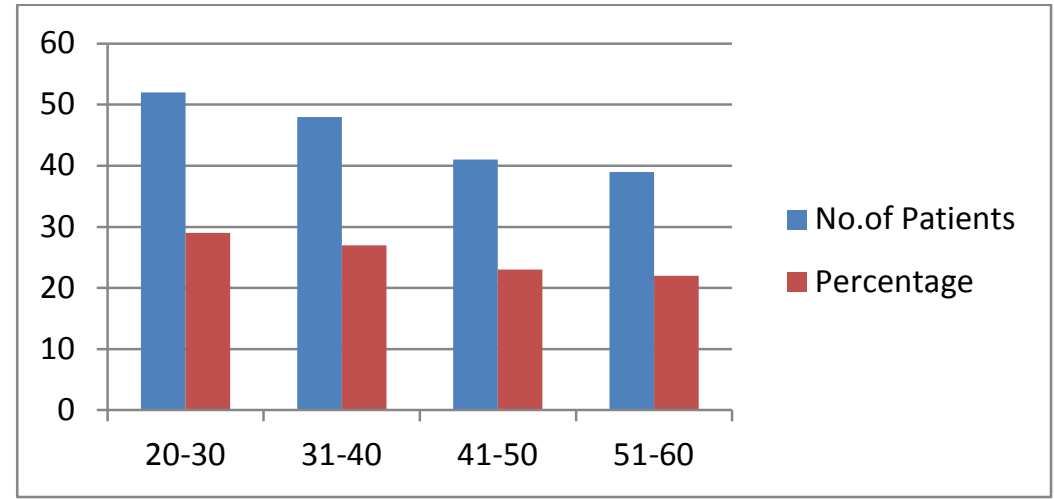

The diagnosis of Rheumatoid Arthritis in the study subjects was based on the following clinical symptoms history of Tender joints 37 (25.1\%) (in $100 \%$ of subjects), swollen joints $(24 \%)$, Joint stiffness $(19.5 \%)$, Fatigue $(16.8 \%)$, and fever $(14.8 \%)$ at the first day of pharmacokinetic assessment.

Table 2

\begin{tabular}{|l|l|}
\hline Symptoms & No. patients (\%) \\
\hline $\begin{array}{l}\text { Tender } \\
\text { joints }\end{array}$ & $37(25.1 \%)$ \\
\hline $\begin{array}{l}\text { swollen } \\
\text { joints }\end{array}$ & $35(23.6 \%)$ \\
Joint & $29(19.5 \%)$ \\
\hline Fatigue & $25(16.8 \%)$ \\
\hline Fever & $22(14.8 \%)$ \\
\hline
\end{tabular}

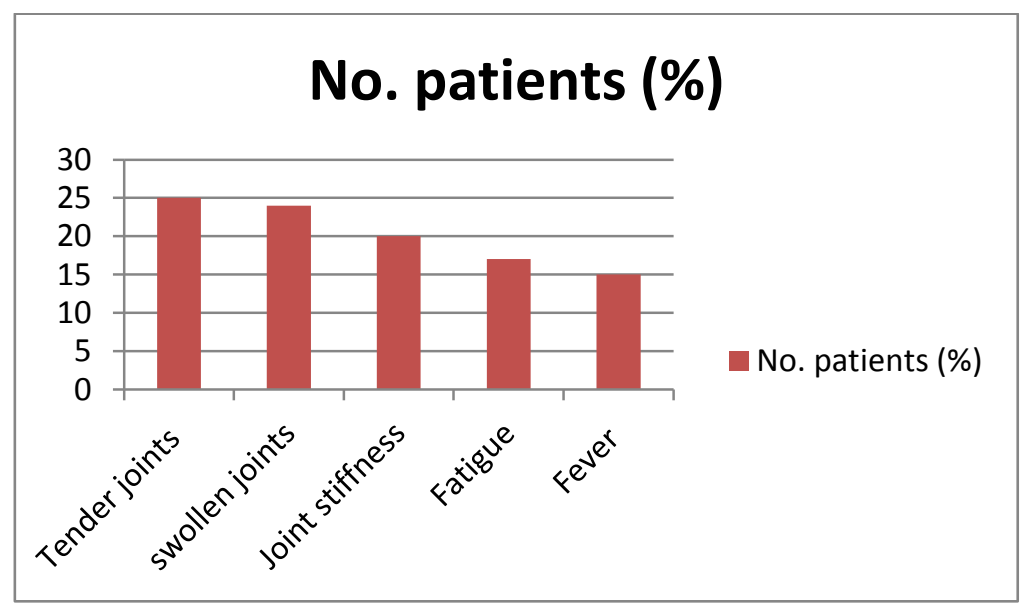




\section{Discussion}

Autoimmunity (tolerance break) develops years before the inflammatory phase of the disease, which can be considered as a golden period for preventing disease progression. Disease-modifying anti rheumatic drugs are the mainstay of therapy, and should be initiated as early as possible in the course of the disease in consultation with a rheumatologist. Rheumatoid Arthritis (RA) is a very heterogeneous disease, the outcome of which is difficult to predict. The vast majority of the patients will have disease progression with bone erosions and cartilage breakdown resulting in joint destruction, functional impairment, and increased mortality.

\section{CONCLUSION}

Glucosamine sulfate might provide some pain relief for people with Rheumatoid Arthritis. Glucosamine has been found to have positive effects on pain treatment \& Relieving pain and improving physical function. MSM may help to relieve joint soreness, stiffness and swelling. MSM is to decrease joint or muscle pain. Collagen peptide significantly reducing pain and joint inflammation. HA control inflammation and synovitis in the foot and ankle joints caused by Rheumatoid Arthritis. Vitamin $\mathrm{C}$ is a powerful antioxidant; it fights molecules that trigger inflammation. Vitamin $\mathrm{C}$ also protects an important protein in your bones and joints.

\section{REFERENCES}

1. Combe B, Progression in early Rheumatoid Arthritis. Best Pract Res Clin Rheumatol, 2009, vol no: 23, Page no: 59-69.

2. Gossec L, Combescure C, Rincheval N, Relative Clinical influence of Clinical, Laboratory, and Radiological Investigations in Early Arthritis on the Diagnosis of Rheumatoid Arthritis. Data from the French Early Arthritis Cohort ESPOIR. J Rheumatol. 2010, vol no: 37, Page no: 2486-92.

3. Finckh A, Liang $M H$, van Herckenrode CM, de Pablo P. Long-term impact of early treatment on radiographic progression in Rheumatoid Arthritis, A meta-analysis, Arthritis Rheum, 2006, vol no: 55, Page no: 864-72.

4. Raza K, et al. Delays in assessment of patients with Rheumatoid Arthritis: variations across Europe. Ann. Rheum, Dis, 2011, vol no: 70, Page no: 1822-1825.

5. Ometto $F$, et al. Methods used to assess remission and low disease activity in Rheumatoid Arthritis, Autoimmun, Rev, 2010, vol no: 9, Page no:161-164. 
6. Grennan DM, Gray J, Loudon J, Fear S. Methotrexate and early postoperative complications in patients with Rheumatoid Arthritis undergoing elective orthopaedic surgery, Ann. Rheum, Dis. 2001, vol no: 60, Page no: 214-217.

7. Nishimura K, et al. Meta-analysis: diagnostic accuracy of anti-cyclic citrullinated peptide antibody and rheumatoid factor for Rheumatoid Arthritis, Ann, Intern. Med. 2007, vol no: 146, Page no: 797-808.

8. Bizzaro $\mathrm{N}$, et al. Anti-cyclic citrullinated peptide antibody titer predicts time to Rheumatoid Arthritis onset in patients with undifferentiated arthritis: results from a 2-year prospective study, Arthritis Res, Ther, 2013, vol no: 15, Page no: R16.

9. Malmstrom V, Catrina AI, Klareskog $L$, The immunopathogenesis of seropositive Rheumatoid Arthritis, from triggering to targeting, Nat. Rev, Immunol, 2017, vol no: 17, Page no: $60-75$.

10. Scott DL. Early Rheumatoid Arthritis. British Medical Bulletin, 2007,Page no: 97-114.

11. Goekoop-Ruiterman YP, de Vries-Bouwstra JK, Allaart CF,Comparison of treatment strategies in early Rheumatoid Arthritis, a randomized trial, Ann Intern Med, 2007, vol no: 146, Page no: 406-15

12. van der Helm-van Mil AH, Detert J, le Cessie S, Validation of a prediction rule for disease outcome in patients with recent - onset undifferentiated arthritis, moving toward individualized treatment decision - making, Arthritis Rheum, 2008, vol no: 58, Page no: 2241-7

13. Finckh A. Early inflammatory arthritis versus Rheumatoid Arthritis. Curr Opin Rheumatol. 2009, vol no: 21, Page no: 118-23.

14. Arnett FC, Edworthy SM, Bloch DA, The American Rheumatism Association 1987 revised criteria for the classification of Rheumatoid Arthritis, Arthritis Rheum, 1988, vol no: 31, Page no: 315-324.

15. van Dongen $\mathrm{H}$, Efficacy of methotrexate treatment in patients with probable Rheumatoid Arthritis: a double-blind, randomized, placebo-controlled trial, Arthritis Rheum, 2007, vol no: 56, Page no: $1424-1432$.

16. Sellam J, B cell activation biomarkers as predictive factors for the response to rituximab in Rheumatoid Arthritis: a six-month, national, multicenter, open-label study, Arthritis Rheum, 2011, vol no: 63, Page no: 933-938. 
17. Seegobin SD, ACPA-positive and ACPA-negative Rheumatoid Arthritis differ in their requirements for combination DMARDs and corticosteroids: secondary analysis of a randomized controlled trial, Arthritis Res, Ther, 2014, vol no: 16, Page no: R13.

18. Raychaudhuri S, Five amino acids in three HLA proteins explain most of the association between MHC and seropositive Rheumatoid Arthritis, Nat, Genet, 2012, vol no: 44, Page no: 291-296.

19. Okada Y, Risk for ACPA-positive Rheumatoid Arthritis is driven by shared HLA amino acid polymorphisms in Asian and European populations. Hum. Mol. Genet, 2014, vol no: 23, Page no: 6916-6926.

20. Mori M, Yamada R, Kobayashi K, Kawaida R, Yamamoto K. Ethnic differences in allele frequency of autoimmune-disease-associated SNPs. J. Hum. Genet, 2005, vol no: 50, Page no: 264-266. 